\section{Sarcopenia: nueva alternativa para el diagnóstico en lugares con acceso limitado a las tecnologías sanitarias}

\section{Sarcopenia: new alternative for diagnosis in places with limited access to health technologies}

Diego Chambergo-Michilot

Red de Eficacia Clínica y Sanitaria (REDECS). Lima. Perú. Universidad Científica del Sur. Lima. Perú.

Correo electrónico: diegochambergomichilot@hotmail.com

Recibido el 26 de septiembre de 2019; aceptado el 29 de octubre de 2019

\section{Sr. Director:}

La sarcopenia es la atrofia muscular asociada al envejecimiento y a las enfermedades avanzadas ${ }^{1}$. Esta enfermedad tiene un gran impacto en la salud porque genera dependencia funcional, lo que aumenta el riesgo de muerte. Se ha estimado que la prevalencia en adultos mayores puede ser mayor del $24 \%{ }^{2}$. Una reciente revisión sistemática informó de que los trastornos lipídicos y la resistencia a la insulina son factores de riesgo para la sarcopenia $^{3}$. Otros factores son la falta de ejercicio, el tabaquismo, el consumo de aminoácidos de cadena ramificada y la baja salud autopercibida ${ }^{4}$.

El grupo EWGSOP2 publicó en 2018 el consenso europeo sobre el diagnóstico de sarcopenia confirmada, definiendo un estado confirmado de esta enfermedad cuando existe poca fuerza muscular y baja cantidad o calidad' Sin embargo, el diagnóstico requiere el uso de tecnologías sanitarias, como la tomografía computarizada, la resonancia magnética o la absorciometría de rayos X de doble energía. Si esas tecnologías no están disponibles, solo se podría diagnosticar la sarcopenia probable, ya que únicamente podrían usarse herramientas de los criterios de baja fuerza muscular (dinamometría, prueba de soporte de la silla y cuestionario SARC-F), que son más económicos.

El diagnóstico se complica en los países en vías de desarrollo, donde el sistema de salud no ha alcanzado una descentralización completa, y el acceso a la salud no es universal. Además, el envejecimiento se ha incrementado en las zonas rurales ${ }^{6}$, lo que junto con la falta de acceso a los servicios de salud en estas áreas contribuye a una mayor prevalencia de sarcopenia no diagnosticada. Se ha demostrado que la hipoxemia podría ser un factor contribuyente para el desarrollo de la sarcopenia ${ }^{7}$, por lo que su incidencia puede aumentar en comunidades de altura. En estos lugares es necesario su diagnóstico, pero el acceso limitado a las tecnologías sanitarias lo limita.
Varios estudios han analizado la capacidad diagnóstica de la combinación de SARC-F positivo ( $\geq 4$ puntos) y la disminución de la circunferencia de la pantorrilla, ya que este último es un proxy para la reducción de la masa muscular ${ }^{5}$. Bahat $\mathrm{G}$ et $\mathrm{al}^{8}{ }^{8}$ informaron de que la especificidad de la combinación de un SARC-F positivo y circunferencia de la pantorrilla $<31 \mathrm{~cm}$ para diagnosticar la sarcopenia en adultos mayores fue del 98\%; además, su razón de probabilidad positiva (+ LR) fue mayor que la + LR del SARC-F solo. Por otro lado, el EWGSOP2 recomienda usar un punto de corte de $>15$ segundos (ambos sexos) en el test de la silla para identificar la baja resistencia. Esta es una prueba económica y rápida que consiste en medir el tiempo en que una persona se para y se sienta cinco veces usando solo la fuerza de sus extremidades inferiores. Pinheiro PA et al. ${ }^{9}$ encontraron que por cada segundo que el test de la silla se prolonga, la probabilidad de sarcopenia aumenta un $8 \%$ en mujeres adultas mayores.

El motivo de esta carta es sugerir la aplicación de un nuevo algoritmo para diagnosticar la sarcopenia confirmada en situaciones de falta de la tecnología sugerida por el consenso europeo (fig. 1). Este algoritmo consiste en SARC-F positivo, un test de la silla $>15$ segundos y circunferencia de la pantorrilla $<31 \mathrm{~cm}$ para mejorar la sensibilidad y la especificidad tanto como sea posible. A pesar de la coherencia de esta idea, se necesita evidencia para recomendarla en lugares con poco acceso a tecnologías sanitarias; esta puede ser generada mediante estudios longitudinales que analicen la sensibilidad, especificidad, valor predictivo positivo y valor predictivo negativo del algoritmo

\section{Conflicto de intereses}

El autor declara no tener ningún conflicto de intereses.

\section{Agradecimientos}

A la Sra. María Campos y el Sr. Isidro Chambergo por el constante ánimo.

\section{BIBLIOGRAFÍA}

1. Han A, Bokshan S, Marcaccio S, DePasse J, Daniels A. Diagnostic criteria and clinical outcomes in sarcopenia research: a literature review. J Clin Med. 2018;7(4):70.

2. III LJM, Khosla S, Crowson CS, O'Connor MK, O'Fallon WM Riggs BL. Epidemiology of sarcopenia. J Am Geriatr Soc. 2000;48(6):625-30

3. Du Y, Oh C, No J. Associations between Sarcopenia and Metabolic Risk Factors: A Systematic Review and Meta-Analysis. J Obes Metab Syndr. 2018;27(3):175.
4. Shimokata H, Ando F. Sarcopenia and its risk factors in epidemiological study. Nihon Ronen Igakkai Zasshi. 2012:49(6):721-5.

5. Cruz-Jentoft AJ, Bahat G, Bauer J, Boirie Y, Bruyère 0, Cederholm $T$, et al. Sarcopenia: revised European consensus on definition and diagnosis. Age Ageing. 2018;48(1):16-31.

6. Wu C, Chen K, Hou M, Chang Y, Chang C, Liu P, et al. Prevalence and associated factors of sarcopenia and severe sarcopenia in older Taiwanese living in rural community: The Tianliao Old People study 04. Geriatr Gerontol Int. 2014;14:69-75.
7. Ng TP, Lu Y, Choo RWM, Tan CTY, Nyunt MSZ, Gao Q, et al. Dysregulated homeostatic pathways in sarcopenia among frail older adults. Aging Cell. 2018;17(6):e12842

8. Bahat G, Oren M, Yilmaz O, Kilic C, Aydin K, Karan M. Comparing SARC-F with SARC-CalF to screen sarcopenia in community living older adults. J Nutr Health Aging. 2018;22(9):1034-8.

9. Pinheiro PA, Carneiro J, Coqueiro R, Pereira R, Fernandes M. "Chair stand test" as simple tool for sarcopenia screening in elderly women. J Nutr Health Aging. 2016;20(1):56-9. 


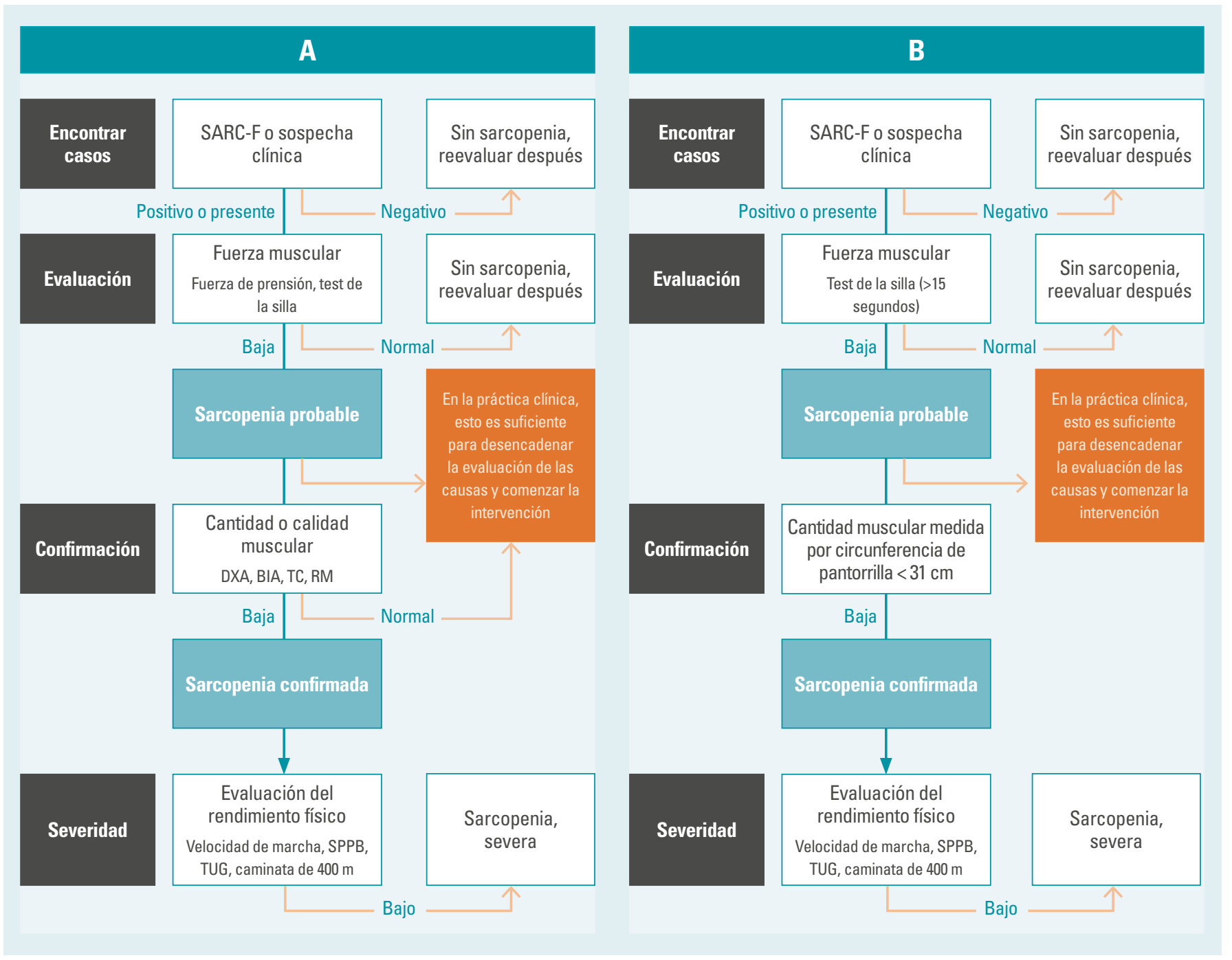

Figura 1. A) Algoritmo de EWGSOP2 para el diagnóstico de sarcopenia confirmada. Adaptado de: Cruz-Jentoft et al. ${ }^{5}$. B) Algoritmo propuesto.

BIA: análisis de bioimpedancia eléctrica; DXA: absorciometría de rayos X de doble energía; RM: resonancia magnética; SPPB: Short Physical Performance Battery, TC: tomografía computarizada; TUG: timed-up-and-go test. 